\title{
An antiaging skin care system containing alpha hydroxy acids and vitamins improves the biomechanical parameters of facial skin
}

This article was published in the following Dove Press journal:

Clinical, Cosmetic and Investigational Dermatology

19 December 2014

Number of times this article has been viewed

\section{Diana Tran \\ Joshua P Townley \\ Tanya M Barnes \\ Kerryn A Greive}

Ego Pharmaceuticals, Braeside, Victoria, Australia
Correspondence: Kerryn A Greive Ego Pharmaceuticals Pty Ltd,

2I-3I Malcolm Road, Braeside,

VIC 3195, Australia

Tel +6I 395868874

Fax +6I 395807647

Email kerryng@egopharm.com
Background: The demand for antiaging products has dramatically increased in recent years, driven by an aging population seeking to maintain the appearance of youth. This study investigates the effects of an antiaging skin care system containing alpha hydroxy acids (AHAs) in conjunction with vitamins $\mathrm{B}_{3}, \mathrm{C}$, and $\mathrm{E}$ on the biomechanical parameters of facial skin.

Methods: Fifty two volunteers followed an antiaging skin care regimen comprising of cleanser, eye cream, day moisturizer, and night moisturizer for 21 days. Wrinkle depth $\left(R_{y}\right)$ and skin roughness $\left(R_{a}\right)$ were measured by skin surface profilometry of the crow's feet area, and skin elasticity parameters $R 2$ (gross elasticity), $R 5$ (net elasticity), $R 6$ (viscoelastic portion), and $R 7$ (recovery after deformation) were determined for facial skin by cutometer, preapplication and after 7, 14, and 21 days. Volunteers also completed a self-assessment questionnaire.

Results: Compared to baseline, $R_{y}$ and $R_{a}$ significantly improved by $32.5 \%(P<0.0001)$ and $42.9 \%$ ( $P<0.0001)$, respectively, after 21 days of antiaging skin care treatment. These results were observed by the volunteers with 9 out of 10 discerning an improvement in skin texture and smoothness. Compared to baseline, $R 2$ and $R 5$ significantly increased by $15.2 \%(P<0.0001)$ and $12.5 \%$ ( $P=0.0449)$, respectively, while $R 6$ significantly decreased by $17.7 \%(P<0.0001)$ after 21 days. $R 7$ increased by $9.7 \%$ after 21 days compared to baseline but this was not significant over this time period.

Conclusion: An antiaging skin care system containing AHAs and vitamins significantly improves the biomechanical parameters of the skin including wrinkles and skin texture, as well as elasticity without significant adverse effects.

Keywords: alpha hydroxy acids, antiaging, nicotinamide, vitamin C, vitamin E, profilometry, cutometer

\section{Introduction}

Skin aging is the result of both intrinsic aging due to the passage of time, and extrinsic aging as a consequence of environmental damage, primarily due to ultraviolet (UV) irradiation. ${ }^{1}$ The fine wrinkles and reduced elasticity which characterize intrinsically aged skin ${ }^{2}$ are exaggerated in photoaged skin, with the development of deep wrinkles and a marked loss of elasticity, as well as dryness, roughness, and dyspigmentation., ${ }^{3,4}$ Wrinkles impair the quality of life for many people due to their perceived unsightly appearance. The demand for cosmeceutical products in the US alone is expected to increase 5.8 percent per annum to $\$ 8.5$ billion in 2015 , driven by an aging population seeking to maintain the appearance of youth. ${ }^{5}$

In the mid-1990s, the antiaging effects of alpha hydroxy acids (AHAs) were recognized by the cosmeceutical industry leading to a proliferation of AHA-containing 
antiaging products. ${ }^{6}$ AHAs are a group of hydrophilic organic acids which contain a carboxylic acid moiety with an adjacent hydroxyl group in the alpha position. ${ }^{7}$ The most commonly used AHAs are glycolic acid and lactic acid, although malic acid, citric acid, pyruvic acid, tartaric acid and others have similar functionality. ${ }^{8}$ The antiaging benefits of AHAs have been known for many years. In ancient times, Cleopatra was said to bathe in sour milk, which contains lactic acid, in order to give her skin a youthful appearance.

The exact mechanism of action for topical AHAs is still unknown, however the most widely accepted theory is that AHAs remove calcium ions from epidermal cell adhesions by chelation. ${ }^{7}$ This results in weakening of the intercellular adhesions which has an exfoliating effect by causing the shedding and flaking of dead and dry cells. ${ }^{7,9}$ The reduced calcium levels also promote further cell growth while slowing cell differentiation, thereby lessening the appearance of wrinkles and making the skin look younger. ${ }^{8}$ AHAs may also promote increased gene expression of collagen and hyaluronic acid in the dermis and epidermis, which in turn improves plumpness and hydration of the skin. ${ }^{9}$

In addition to AHAs, certain vitamins have also been shown to be provide antiaging benefits. Nicotinamide is the water-soluble, active form of vitamin $\mathrm{B}_{3}$. Topical nicotinamide has been shown to improve fine lines and wrinkles, hyperpigmented spots, red blotchiness and sallowness (yellowing), as well as elasticity. ${ }^{10,11}$ In addition, nicotinamide has been demonstrated to increase the skin's production of collagen and ceramides, and to stimulate keratinocyte differentiation, leading to improved barrier function and skin appearance. $^{12}$

Ascorbyl tetraisopalmitate is a unique oil-soluble vitamin $\mathrm{C}$ derivative which has superior percutaneous absorption compared to other vitamin $\mathrm{C}$ derivatives. Vitamin $\mathrm{C}$ is an antioxidant which is required for collagen synthesis. ${ }^{13}$ Topical vitamin $\mathrm{C}$ has been shown to protect the skin from UV induced damage by reducing the amount of free radical formation. ${ }^{13}$ It has also been suggested that vitamin $\mathrm{C}$ reduces wrinkles and may help to repair age-related skin damage. $^{13,14}$

Tocopherol, also known as vitamin E, is an important freeradical quenching, lipid soluble antioxidant. ${ }^{15,16}$ Vitamin $\mathrm{E}$ has been shown to prevent UV-induced skin damage, and can also improve moisture content and barrier function of the skin. ${ }^{16}$ Further, vitamin $\mathrm{E}$ has been demonstrated to reduce fine lines and wrinkles and improve skin smoothness. ${ }^{15,16}$

This study investigates the effects of an antiaging skin care system containing AHAs in conjunction with vitamins $\mathrm{B}_{3}, \mathrm{C}$, and $\mathrm{E}$ in healthy Caucasian women. Specifically, the biomechanical parameters of wrinkle depth $\left(R_{y}\right)$ and skin roughness $\left(R_{a}\right)$ were measured by skin surface profilometry of the crow's feet area, and the skin elasticity parameters $R 2$ (gross elasticity), $R 5$ (net elasticity), $R 6$ (viscoelastic portion), and $R 7$ (recovery after deformation) of facial skin were determined by cutometer preapplication and after 7, 14, and 21 days following the antiaging skin care regimen. Volunteers were also asked to complete a self-assessment questionnaire.

\section{Materials and methods Topical preparations}

The formulations studied were: Elucent Anti-aging Gentle Cleanser (Ego Pharmaceuticals Pty Ltd, VIC, Australia) containing 1\% lactic acid, 1\% glycolic acid, vitamins $\mathrm{B}_{3}$ and $\mathrm{E}$, water, glycerin, decyl glucoside, disodium cocoamphodiacetate, 1,2-hexanediol, caprylyl glycol, xanthan gum, silica, titanium dioxide, tin oxide, panthenol, Avena sativa (oat) kernel extract, and ascorbyl tetraisopalmitate; Elucent Anti-aging Eye Cream (Ego Pharmaceuticals Pty Ltd) containing 4\% glycolic acid, vitamins $\mathrm{B}_{3}, \mathrm{C}$, and E, water, cyclopentasiloxane, glycerin, cetearyl alcohol, sodium PCA, paraffinum liquidum, panthenol, glyceryl stearate, 1,2-hexanediol, caprylyl glycol, cyclotetrasiloxane, PEG-100 stearate, xanthan gum, sodium polyacrylate, A. sativa (oat) kernel extract, disodium ethylenediaminetetraacetic acid (EDTA), and ammonium hydroxide; Elucent Anti-aging Day Moisturizer (Ego Pharmaceuticals Pty Ltd) containing 4\% glycolic acid, vitamins $\mathrm{B}_{3}$ and $\mathrm{E}$, water, dipropyl adipate, cyclotetrasiloxane, isostearic acid, silica, glyceryl stearate, magnesium aluminum silicate, PEG-100 stearate, cetyl alcohol, panthenol, titanium dioxide, 1,2-hexanediol, caprylyl glycol, glycerin, A. sativa (oat) kernel extract, dimethicone, xanthan gum, decyl glucoside, disodium EDTA, iron oxides, propylene glycol, and ammonium hydroxide with a broad spectrum SPF 30+; Elucent Anti-aging Night Moisturizer (Ego Pharmaceuticals Pty Ltd) containing 4\% lactic acid, 4\% glycolic acid, vitamins $\mathrm{B}_{3}$, E and C, water, cyclopentasiloxane, paraffinum liquidum, cyclohexasiloxane, cetyl alcohol, cetearyl olivate, panthenol, sorbitan olivate, glycerin, A. sativa (oat) kernel extract, dimethicone, xanthan gum, sodium polyacrylate, ammonium hydroxide, disodium EDTA, butylphenyl methylpropional, citronellol, hexyl cinnamal, and linalool.

\section{Skin surface profilometry}

Fifty two volunteers aged between 30 years and 55 years were enrolled in the study. Eligible volunteers were: Caucasian 
females aged greater than 30 years; not taking medication or under the care of a doctor for a period of 1 month prior to commencement and throughout the entire test period; completed an extensive medical history form; free of any dermatological or systemic disorder that would interfere with results; had self-described dry skin; available for the study duration, gave written informed consent; prior use of products containing AHAs without any adverse effect; had no adverse effect to a 24-hour patch test screening to the test products. Exclusion criteria were: individuals under doctors care; taking medication which could mask or interfere with test results; history of sensitivity to cosmetics in general and moisturizers in particular; any form of skin cancer or any disease that could interfere with test results; diagnosed with chronic skin allergies; excessive hair on test sites; known hypersensitivity to cosmetic products; females who were pregnant or nursing an infant.

In order to precondition test sites and keep all topical treatments constant for all volunteers, volunteers were required to abstain from using deodorant soaps, moisturizing soaps, or cosmetic moisturizers on the test area for a period of 10 days prior to commencement, and during the course of the study. At the completion of the 10 day period, volunteers returned to the test facility where they were trained how to apply the antiaging skin care products.

Volunteers followed a daily antiaging skin care regimen for 21 days. Each morning the face and neck were dampened with warm water, cleanser was applied and gently massaged to produce a lather after which the skin was rinsed thoroughly and patted dry. A layer of eye cream was then applied around the eye area with gentle patting and day moisturizer was liberally applied to the face and neck. Each night the face and neck were again dampened with warm water, cleanser applied and gently massaged to produce a lather. Skin was then rinsed thoroughly and patted dry. A layer of eye cream was then applied around the eye area with gentle patting and night moisturizer was liberally applied to the face and neck.

To assess changes in the wrinkle area, a single silicone replica was randomly taken of each volunteer's $(n=15)$ right or left crow's feet area at $t=0$ (preapplication) and after 7, 14, and 21 days following the antiaging skin care regimen, and stored at $30^{\circ} \mathrm{C}$ and $90 \%$ relative humidity until analyzed by optical profilometry. ${ }^{17}$

Profilometry was performed using a Surftest SJ-400 Profilometer (Mitutoyo, Kanagawa, Japan). ${ }^{17}$ The horizontal speed of the stylus was $48 \mathrm{~mm} \mathrm{~min}^{-1}$ and data was collected for 5 seconds yielding a scan length of $4 \mathrm{~mm}$. Three replicates were performed of each silicone replica, each no more than $0.5 \mathrm{~mm}$ apart. The vertical displacements of the stylus were plotted versus time. $R_{y}$ (wrinkle depth) represents the maximum peak height of the profile minus the minimum valley of the profile (Figure 1); $R_{y}=$ peak (maximum) - valley (minimum). $R_{a}$ (mean surface roughness) represents the area that the profile deviates above and below an average line generated to run through the center of the profile (Figure 1); $R_{a}=1 / \ell_{0}{ }^{\ell}|y(x)| \mathrm{d} x$, where $\ell$ is the scan length and $|y(x)|$ is the

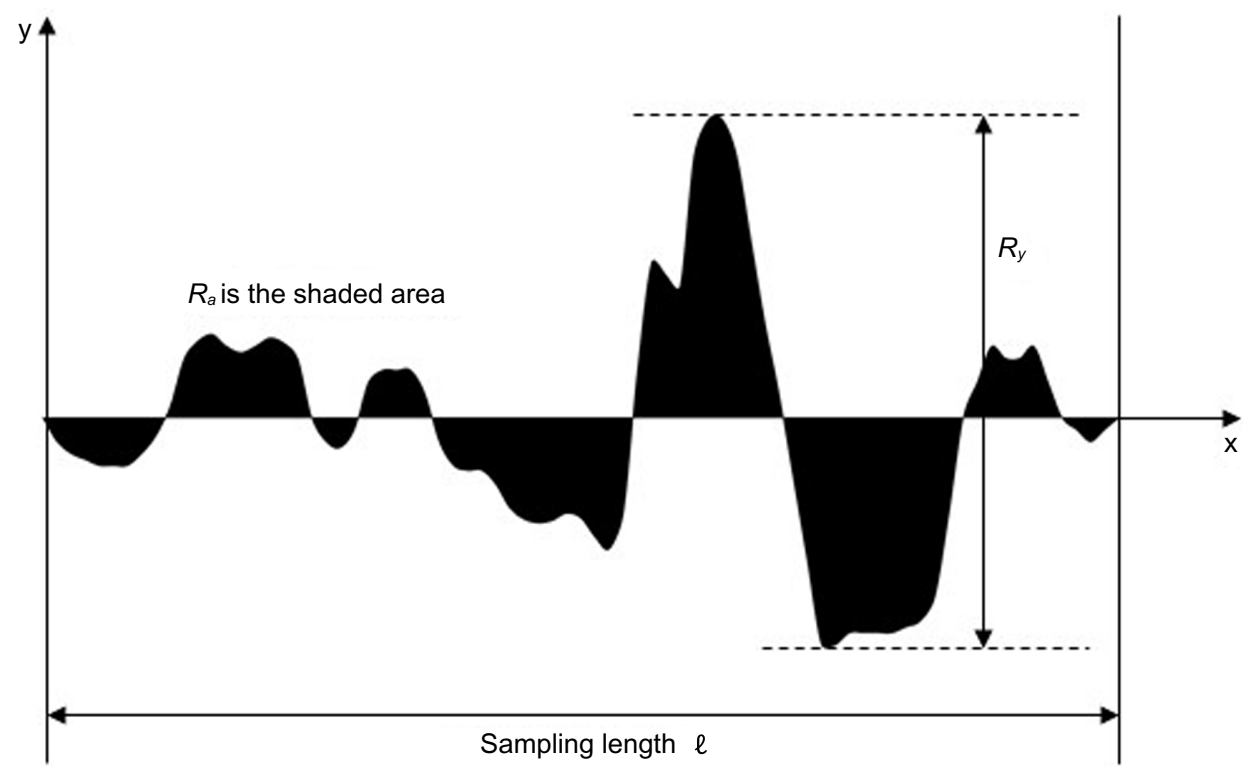

Figure I Skin surface curve obtained with a profilometer depicting $R_{y}$ (wrinkle depth) and $R_{a}$ (mean surface roughness).

Notes: $R_{y}$ represents the maximum peak height of the profile minus the minimum valley of the profile, $R_{y}=$ peak (maximum) - valley (minimum). $R_{a}$ represents the area that the profile deviates above and below an average line generated to run through the center of the profile, $R_{a}=\left|/ \ell_{0} \int^{l}\right| y(x) \mid \mathrm{d} x$, where $\ell$ is the scan length and $|y(x)|$ is the absolute value of the location of the profile relative to the mean profile height. 
absolute value of the location of the profile relative to the mean profile height.

\section{Skin elasticity}

The same volunteers were used for both the skin profilometry and skin elasticity studies. A Cutometer SEM 575 (Courage and Khazaka Electronic GmbH, Cologne, Germany) was used to measure the elasticity of the skin surface. The measuring principle is based on the suction method. ${ }^{18}$ The resistance of the skin to the negative pressure (firmness) and its ability to return into its original position (elasticity) are displayed as curves (Figure 2) in real time during the measurement.

The measuring probe (Mode 1; time/strain) applied a constant suction of 400 bar for 5 seconds followed by a relaxation time of 3 seconds, with one repetition. Before each set of measurements, volunteers were required to equilibrate in a closed environment with a constant temperature $\left(20^{\circ} \mathrm{C} \pm 2{ }^{\circ} \mathrm{C}\right)$ and humidity (55\%-65\% relative humidity). Measurements were taken at $t=0$ (preapplication) and after 7,14 , and 21 days following the antiaging skin care regimen at four different closely adjoining points on the face of each of the 52 volunteers. The cutometer generated a graph depicting immediate deformation or skin extensibility $\left(U_{\mathrm{e}}\right)$, delayed distension $\left(U_{\mathrm{v}}\right)$, final deformation $\left(U_{\mathrm{f}}\right)$, immediate retraction $\left(U_{\mathrm{r}}\right)$, total recovery $\left(U_{\mathrm{a}}\right)$, and residual deformation at the end of measuring cycle (R) (Figure 2). The mechanical parameters $R 2, R 5$, $R 6$, and $R 7$ were subsequently calculated.

$R 2$ refers to the gross elasticity of the skin including the viscous deformation, and is represented by the ratio of "the ability of redeformation of skin" to "final distension": $R 2=U_{\mathrm{a}} / U_{\mathrm{f}^{\circ}}{ }^{19}$ The closer the value is to $1(100 \%)$, the more elastic the skin is.

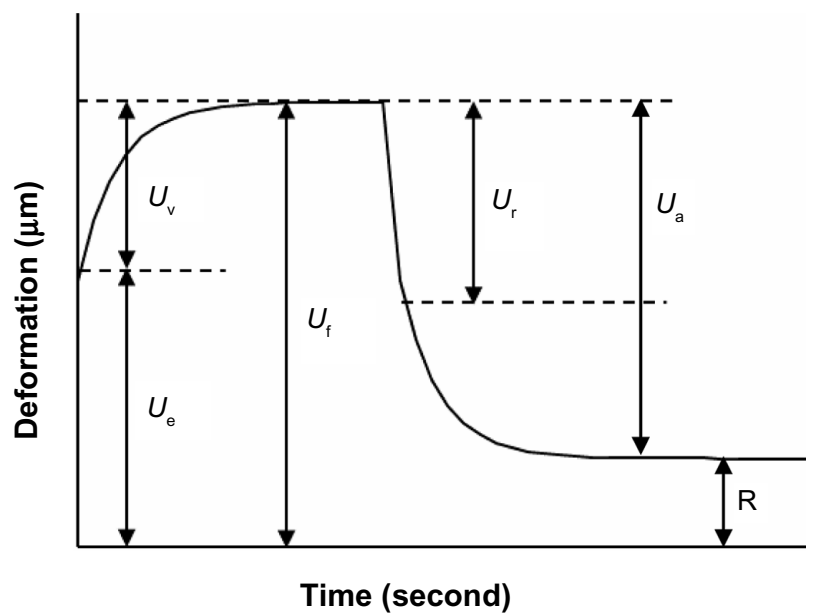

Figure 2 Skin deformation curve obtained with a cutometer depicting the immediate deformation or skin extensibility $\left(U_{e}\right)$, delayed distension $\left(U_{v}\right)$, final deformation $\left(U_{f}\right)$, immediate retraction $\left(U_{r}\right)$, total recovery $\left(U_{\mathrm{a}}\right)$, and residual deformation at the end of the measuring cycle $(\mathrm{R})$.
$R 5$ refers to the net elasticity of the skin without the viscous deformation and is represented by the ratio of "the immediate retraction" to "immediate distension": $R 5=U_{\mathrm{r}} / U_{\mathrm{e}} \cdot{ }^{19}$ The closer the value is to $1(100 \%)$, the more elastic the skin is. $R 6$ represents the portion of the viscoelasticity on the elastic part of the curve, and is represented by the ratio of "viscoelastic" to "elastic distension": $R 6=U_{\mathrm{v}} / U_{\mathrm{e}} \cdot{ }^{19}$ Since the $R 6$ parameter measures the stretch capacity of the skin, negative values reflect improved skin condition. $R 7$ refers to recovery after deformation. It is the portion of elasticity compared to the final distension. It is represented by the ratio of "the immediate retraction" to "final distension" $R 7=U_{\mathrm{r}} / U_{\mathrm{f}}{ }^{19}$ The closer the value is to $1(100 \%)$, the more elastic the skin is.

\section{Self-assessment questionnaire}

Subject self-assessments (Table 1) were made after 7, 14, and 21 days following the antiaging skin care regimen.

\section{Statistical analysis}

The percentage changes for individual values of different parameters was determined by the following equation: Percentage change $=[(a-b) / b] \times 100$, where " $a$ " is the individual value of $R_{y}, R_{a}, R 2, R 5, R 6$, or $R 7$ at each individual time point and " $b$ " is the corresponding zero-time value. The Student's $t$-test was used to compare the mean values and $\%$ change at each time point compared to preapplication. $P<0.05$ was considered statistically significant.

\section{Results}

\section{Skin surface profilometry}

Fifty two volunteers completed the study with an average age of $42.4 \pm 1.3$ years. Just over one-quarter of the volunteers reported a mild transient burning/tingling sensation upon application of products at day 7 , which decreased with continued use. Fifteen volunteers were randomly selected for skin surface profilometry of the crow's feet area. Table 2 shows the average values for $R_{y}$ (wrinkle depth) and $R_{a}$ (average skin roughness) over time. All 15 volunteers showed significant reduction in both parameters compared to baseline. Figure 3 illustrates the percentage reduction in $R_{y}$ and $R_{a}$ over time. Wrinkle depth $\left(R_{y}\right)$ significantly reduced by $16.4 \%(P=0.0007$ versus $t=0)$ after 7 days of antiaging skin care treatment, and continued to reduce by $24.1 \%(P<0.0001$ versus $t=0)$ after 14 days and $32.5 \%(P<0.0001$ versus $t=0)$ after 21 days. Similarly, average skin roughness $\left(R_{a}\right)$ significantly reduced by $20.9 \%(P=0.0004$ versus $t=0)$ after 7 days, and continued to reduce by $33.8 \%(P<0.0001$ versus $t=0)$ after 14 days and $42.9 \%(P<0.0001$ versus $t=0)$ after 21 days. 
Table I Self-assessment questionnaire showing the percentage of positive responses from volunteers following an antiaging skin care regimen for 7,14 , and 21 days $(n=52)$

\begin{tabular}{|c|c|c|c|}
\hline Question & 7 days & 14 days & 2I days \\
\hline Have you seen a reduction in fine lines? & 40.4 & 67.3 & 73.1 \\
\hline Have you seen a reduction in skin pigmentation? & 32.7 & 46.2 & 55.8 \\
\hline Have you seen an improvement in the skin firmness of your neck? & 57.7 & 67.3 & 75.0 \\
\hline Have you seen a reduction in fine lines around your eyes? & 40.4 & 67.3 & 73.1 \\
\hline Have you seen a reduction in wrinkles? & 11.5 & 50.0 & 57.7 \\
\hline Does your skin look rejuvenated? & 78.9 & 82.7 & 90.4 \\
\hline Have you seen an improvement in the skin elasticity of your neck? & 57.7 & 71.2 & 76.9 \\
\hline Have you seen a reduction in wrinkles around your mouth? & 13.5 & 34.6 & 46.2 \\
\hline Does your skin look younger? & 59.6 & 63.5 & 76.9 \\
\hline Have you seen a reduction in "bags" around your eyes? & 34.6 & 55.8 & 57.7 \\
\hline Does your skin look regenerated? & 75.0 & 84.6 & 88.5 \\
\hline Do you look more refreshed? & 80.8 & 86.5 & 86.5 \\
\hline Have you seen a reduction in fine lines around your mouth? & 30.8 & 46.2 & 53.9 \\
\hline Have you seen an improvement in skin appearance around your eyes? & 50.0 & 76.5 & 82.7 \\
\hline Do you look less tired? & 57.7 & 71.2 & 80.77 \\
\hline Have you seen an improvement in skin tone? & 57.7 & 76.9 & 84.6 \\
\hline Have you seen a reduction in skin roughness? & 78.9 & 84.6 & 88.5 \\
\hline Have you seen an improvement in skin elasticity? & 69.2 & 76.9 & 84.6 \\
\hline Does your skin feel rejuvenated? & 78.4 & 86.5 & 88.5 \\
\hline Have you seen an improvement in skin firmness? & 67.3 & 80.8 & 84.6 \\
\hline Do you feel younger? & 46.2 & 55.8 & 57.7 \\
\hline Have you seen an improvement in the skin tone of your neck? & 46.2 & 69.2 & 75.0 \\
\hline Does your skin look more radiant? & 78.9 & 86.3 & 88.2 \\
\hline Have you seen a reduction in wrinkles around your eyes? & 17.3 & 51.9 & 65.4 \\
\hline Have you seen an improvement in skin clarity? & 73.1 & 82.4 & 88.5 \\
\hline Have you seen an improvement in the skin radiance of your neck? & 50.0 & 64.6 & 75.5 \\
\hline Did you like the texture of each product? & 86.3 & 86.5 & 86.5 \\
\hline Do you feel more refreshed? & 75.0 & 82.7 & 86.5 \\
\hline Do you feel more beautiful after regular use of the range? & 65.4 & 72.6 & 75.0 \\
\hline Have you seen an improvement in skin softness? & 82.7 & 92.3 & 92.3 \\
\hline Have you seen a reduction in skin redness? & 36.5 & 48.1 & 53.9 \\
\hline Would you recommend this range to your friends? & 68.6 & 82.7 & 78.9 \\
\hline Do you look younger? & 48.1 & 61.5 & 71.2 \\
\hline Have you seen an improvement in skin texture? & 80.8 & 86.5 & 86.5 \\
\hline Is your complexion more youthful? & 55.8 & 69.2 & 76.9 \\
\hline Have you seen a reduction in puffiness around your eyes? & 36.5 & 55.8 & 61.5 \\
\hline Have friends commented on the appearance of your skin? & 30.8 & 50.0 & 57.7 \\
\hline Have you seen a reduction in skin coarseness? & 71.2 & 86.5 & 88.5 \\
\hline Does your skin feel regenerated? & 80.4 & 86.5 & 90.2 \\
\hline Have you seen a reduction in dark circles around your eyes? & 31.4 & 44.2 & 60.0 \\
\hline Does your skin feel rehydrated? & 86.3 & 92.3 & 92.3 \\
\hline Would you continue to use this range if able to? & 88.5 & 90.4 & 88.5 \\
\hline Have you seen an improvement in skin smoothness? & 84.6 & 90.4 & 90.2 \\
\hline
\end{tabular}

Note: Relevant performance questions pertaining to skin texture and smoothness are highlighted.

\section{Skin elasticity}

Table 3 shows the average skin elasticity parameters $R 2$ (gross elasticity), $R 5$ (net elasticity), $R 6$ (viscoelastic portion), and $R 7$ (recovery after deformation) over time for facial skin of all 52 volunteers. Figure 4 illustrates the percentage increase in $R 2, R 5$, and $R 7$, and percentage decrease in $R 6$ over time compared to baseline. $R 2$ significantly increased by $7.5 \%$ $(P<0.0001$ versus $t=0)$ after 7 days of antiaging skin care treatment, and continued to improve by $10.2 \%(P<0.0001$ versus $t=0)$ after 14 days and $15.2 \%(P<0.0001$ versus $t=0)$ after 21 days. Similarly, $R 5$ increased by $9.1 \%$ after 7 days ( $P=0.0055$ versus $t=0$ ), and continued to improve by $11.0 \%$ $(P=0.0072$ versus $t=0)$ after 14 days and $12.5 \%(P=0.0449$ versus $t=0)$ after 21 days. $R 6$ was unchanged, $-0.02 \%$ ( $P=0.5089$ versus $t=0)$, after 7 days, but significantly decreased by $6.9 \%(P=0.0026$ versus $t=0)$ after 14 days, and $17.7 \%(P<0.0001$ versus $t=0)$ after 21 days. $R 7$ increased by $6.2 \%, 11.3 \%$, and $9.7 \%$ after 7,14 , and 21 days, respectively, 
Table 2 Characterization of skin surface topography of the crow's feet area antiaging

\begin{tabular}{lll}
\hline $\begin{array}{l}\text { Time } \\
\text { (days) }\end{array}$ & $\begin{array}{l}\boldsymbol{R}_{y}(\text { wrinkle } \\
\text { depth) }(\mu \mathrm{m})\end{array}$ & $\begin{array}{l}\boldsymbol{R}_{a} \text { (average skin } \\
\text { roughness) }(\mu \mathrm{m})\end{array}$ \\
\hline 0 & $37.2 \pm 2.2$ & $7.4 \pm 0.6$ \\
7 & $30.9 \pm 2.1^{* *}$ & $5.7 \pm 0.4^{* *}$ \\
14 & $27.9 \pm 1.9^{*}$ & $4.8 \pm 0.4^{*}$ \\
21 & $25.1 \pm 2.1^{*}$ & $4.1 \pm 0.4^{*}$ \\
\hline
\end{tabular}

Notes: Parameters $R_{y}$ (wrinkle depth; $\mu \mathrm{m}$ ) and $R_{a}$ (average skin roughness; $\mu \mathrm{m}$ ) were measured by skin surface profilometry at day 0 (pre-application), and following a daily antiaging skin care regimen for 7,14 , and 21 days. Results are presented as mean \pm SEM. $* P<0.000$ I versus $t=0$; $* * P<0.00$ I versus $t=0$.

however, this increase was only significant after 14 days $(P=0.0334$ versus $t=0)$ and not after $7(P=0.1592$ versus $t=0)$ or $21(P=0.0933$ versus $t=0)$ days.

\section{Self-assessment questionnaire}

All 52 volunteers completed a self-assessment questionnaire involving 44 yes/no responses at days 7, 14, and 21 (Table 1). Four relevant performance questions pertaining to skin texture and smoothness were included and are highlighted in Table 1. $89 \%$ volunteers observed a reduction in skin roughness and skin coarseness, while $87 \%$ volunteers observed an improvement in skin texture and $90 \%$ observed an improvement in skin smoothness after 21 days following the antiaging skin care treatment.

\section{Discussion}

Exposure of human skin to UV irradiation leads to physiological and biochemical changes. ${ }^{1}$ Photoaged skin is clinically characterized by wrinkles, dryness, inelasticity and irregular pigmentation. ${ }^{3,4}$ In order to try and reverse these signs of aging, consumers are faced with an ever increasing number

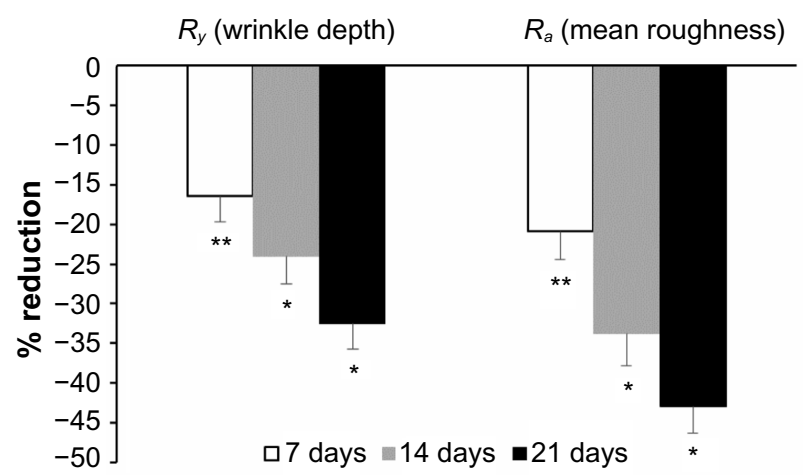

Figure 3 The percentage reduction in $R_{y}$ (wrinkle depth) and $R_{a}$ (mean surface roughness) versus time (days).

Notes: The reduction was determined by skin surface profilometry for the crow's feet area of volunteers following an antiaging skin care regimen for 21 days $(n=15)$. Results are presented as mean $\pm S E M$. $* P<0.000$ I versus $t=0$, $* * P<0.001$ versus $t=0$. Abbreviation: SEM, standard error of mean.
Table 3 Characterization of facial skin elasticity antiaging

\begin{tabular}{lllll}
\hline $\begin{array}{l}\text { Time } \\
\text { (days) }\end{array}$ & R2 & R5 & R6 & $R 7$ \\
\hline 0 & $0.645 \pm 0.014$ & $0.416 \pm 0.015$ & $0.314 \pm 0.007$ & $0.326 \pm 0.011$ \\
7 & $0.683 \pm 0.01 I^{*}$ & $0.444 \pm 0.013^{* * *}$ & $0.310 \pm 0.006$ & $0.338 \pm 0.009$ \\
14 & $0.696 \pm 0.00 I^{*}$ & $0.447 \pm 0.010^{* *}$ & $0.287 \pm 0.007^{* *}$ & $0.348 \pm 0.007^{* *}$ \\
21 & $0.726 \pm 0.008^{*}$ & $0.446 \pm 0.01 I^{* *}$ & $0.254 \pm 0.005^{*}$ & $0.344 \pm 0.008$ \\
\hline
\end{tabular}

Notes: Parameters R2 (gross elasticity), R5 (net elasticity), R6 (viscoelastic portion) and $R 7$ (recovery after deformation) (arbitrary units) were calculated by using cutometer at day 0 (pre-application), and following a daily antiaging skin care regimen for 7,14 , and 21 days Results are presented as mean \pm SEM. $* P<0.000$ I versus $t=0 ; * * P<0.05$ versus $t=0$.

of over-the-counter (OTC) products, many of which have undergone little to no testing of their efficacy. In Australia, consumer products which are available OTC usually contain up to $20 \%$ AHAs, however higher concentrations may be used by doctors, dermatologists, or in salons by trained professionals..$^{20}$ In this study the biomechanical effects of an antiaging skin care system containing up to $8 \%$ AHAs as well as vitamins $\mathrm{B}_{3}, \mathrm{C}$, and $\mathrm{E}$ was examined, although products containing up to $16 \%$ AHAs are available in this range.

Characterization of skin surface topography demonstrated a significant improvement compared to baseline of $16.4 \%$ for wrinkle depth $\left(R_{y}\right)$ of the crow's feet area after only 7 days following the antiaging skin care regimen, which continued to significantly increase to $32.5 \%$ improvement after 21 days. Surface roughness $\left(R_{a}\right)$ also significantly improved compared to baseline by $20.9 \%$ after only 7 days, to $42.9 \%$ after 21 days. These results were observed by volunteers, with 9 out of 10 women discerning changes in skin texture and smoothness.

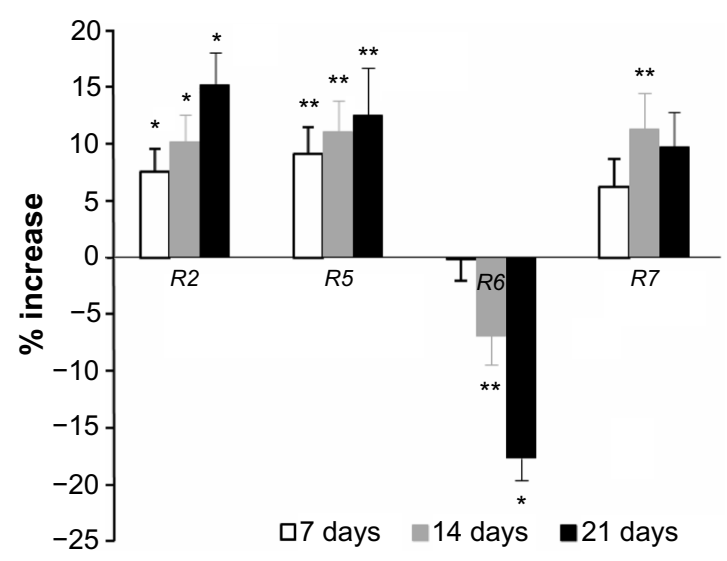

Figure 4 The percentage increase in skin elasticity parameters $R 2$ (gross elasticity), R5 (net elasticity), R6 (viscoelastic portion), and R7 (recovery after deformation) versus time (days).

Notes: The increase was determined by cutometer for facial skin of volunteers following an antiaging skin care regimen for 21 days $(n=52)$. Results are presented as mean \pm SEM. $* P<0.000$ I versus $t=0$, $* * P<0.05$ versus $t=0$.

Abbreviation: SEM, standard error of mean. 
Improvements in skin elasticity of facial skin were also found in this study compared to baseline measurements. Specifically, gross elasticity $(R 2)$ was significantly improved by $7.5 \%$ after 7 days, and continued to significantly improve by $15.2 \%$ after 21 days. $R 2$ measures the ability of the skin to return to its original position after deformation and is related to the function of elastic fibers of the skin. ${ }^{21}$ Net elasticity (R5) was also significantly improved by $9.1 \%$ after 7 days, and continued to significantly improve by $12.5 \%$ after 21 days. $R 5$ is the parameter of choice for quantifying skin aging as it represents the elastic recovery (ability of the skin to recover after deformation) independent of skin thickness. ${ }^{22}$ Further, the viscoelastic portion $(R \sigma)$ was unchanged after 7 days, but was significantly decreased by $17.7 \%$ after 21 days. $R 6$ indicates the relative contribution of viscoelastic plus viscous deformation to elastic deformation of the skin and is attributed to a decrease in the viscosity of interstitial fluid as a result of increased water content and changes in proteoglycan composition and/or structure. The accumulation of water in the dermis diminishes the friction between fibers and facilitates movement of interstitial fluid. ${ }^{23}$ Although the increase in recovery after deformation $(R 7)$ of $9.7 \%$ did not reach significance, there was a clear trend which would be expected to reach significance in time.

Formulations containing $>20 \%$ AHAs have been used in clinical practice for decades to treat a variety of skin conditions including acne, ichthyosis, keratoses, warts, and psoriasis, and it's efficacy at this concentration in photoaged skin has been reported in a large number of published studies. ${ }^{24-27}$ These formulations are not available OTC, unlike the formulations used in this study. To date, there have only been three clinical studies published showing the effects of AHAs on photoaging at low concentrations, similar to those used in this study. When applied at $8 \%$ (glycolic acid or L-lactic acid) for 22 weeks, the majority of patients ( $76 \%$ for glycolic acid; $71 \%$ for lactic acid) reported a noticeable improvement in the appearance and smoothness of photoaged skin. ${ }^{28}$ Treatment with $12 \%$ lactic acid for 3 months has been shown to result in increased epidermal and dermal firmness and thickness, and clinical improvement in skin smoothness and the appearance of lines and wrinkles. ${ }^{29}$ No dermal changes were observed after treatment with $5 \%$ lactic acid, however, similar clinical and epidermal changes were noted ${ }^{29}$ Further, application of $5 \%$ glycolic acid cream for 3 months has been shown to improve skin texture and discoloration of photoaged skin..$^{30}$ To date there have been no published clinical studies on the effects of AHAs and vitamins on photoaging.
The benefits of AHAs in the antiaging skin system investigated in this study is complemented by the additive effects of vitamin $B_{3}$, for improved skin barrier function and appearance, ${ }^{12}$ Vitamin $C$, to help repair age-related damage ${ }^{14}$ and Vitamin E, for minimizing free-radical-induced damage. ${ }^{16}$ All products in the range are free of traditional preservatives, alcohol and soap to reduce their irritation potential. Further, the products are noncomedogenic, nonirritating and nonsensitizing to the skin as determined by repeat insult patch tests, and nonirritating to the eyes (data not shown). In addition, the day moisturizer has a broad spectrum SPF of $30+$ as sun protection is essential when using a product containing AHAs as these ingredients increase the skin's sensitivity to UV light.

With such high demand, it is no surprise that there are a multitude of antiaging products on the market, making it vital that consumers are aware of what they are buying. No matter what ingredients are used, the efficacy of commercially available OTC antiaging products should be demonstrated, ${ }^{8}$ otherwise consumers are simply buying "hope in a jar". For instance, while the commonly used AHAs glycolic acid and lactic acid possess similar efficacy, there may be significant differences in efficacy between retinoids, another popular antiaging ingredient, although all can be claimed as Vitamin A on the product label. For example, retinol lotion has been shown to significantly improve wrinkles, ${ }^{31}$ and synthetic retinyl- $N$-formyl aspartame has also been demonstrated to improve skin roughness and wrinkles ${ }^{31}$ However, studies of retinyl esters, such as retinyl palmitate and retinyl propionate fail to show good efficacy. ${ }^{31}$ The most promising retinoid appears to be retinaldehyde, with application resulting in a significant reduction of wrinkles and surface roughness. ${ }^{32}$ However, although promising, the efficacy of retinaldehyde is yet to be supported by clinical trials, with only in vitro studies and small scale human trials conducted.$^{31}$ Further, tretinoin (retinoic acid or vitamin A acid), the biologically active form, which has been extensively studied and is known to markedly improve the complexion and appearance of wrinkles, is classed as a drug and is not used in OTC products. ${ }^{20}$

Minimal side effects were observed in this study with just over one-quarter of volunteers reporting a mild transient burning/tingling sensation upon application of products at day 7 , which decreased with continued use. This has been observed by others with AHA levels at cosmetic concentrations. ${ }^{20}$ Side effects of AHA treatment have been shown in other studies, albeit at higher AHA concentrations, to include dryness, a burning sensation, erythema, or photosensitization, however, studies have shown that AHAs are better tolerated 
that retinoids. ${ }^{33}$ For example, one questionnaire found that patients receiving topical glycolic acid, who had previously received topical tretinoin preferred treatment with glycolic acid. $^{26}$

In conclusion, this is the first study to demonstrate that an antiaging skin care system containing AHAs and vitamins significantly improves the biomechanical parameters of the skin including wrinkles and skin texture, as well as elasticity without any significant adverse effects over 21 days compared to baseline measurements. Further improvement of these parameters would be expected with continued use.

\section{Acknowledgments}

The authors would like to thank John Staton, Dermatest Pty Ltd for assistance in performing these studies.

\section{Disclosure}

Diana Tran, Joshua P Townley, Tanya M Barnes and Kerryn A Greive are employed by Ego Pharmaceuticals, the sponsor of the study and manufacturer of the Elucent range. The authors report no other conflicts of interest in this work.

\section{References}

1. Langton AK, Sherratt MJ, Griffiths CEM, Watson REB. A new wrinkle on old skin: the role of elastic fibres in skin ageing. Int J Cosmet Sci. 2010;32(5):330-339.

2. Montagna W, Kirchner S, Carlisle K. Histology of sun-damaged humanskin. JAm Acad Dermatol. 1989;21(5 Pt 1):907-918.

3. Warren R, Gartstein V, Kligman AM, et al. Age, sunlight, and facial skin: a histologic and quantitative study. J Am Acad Dermatol. 1991; 25(5 Pt 1):751-760.

4. Rokhsar CK, Lee S, Fitzpatrick RE. Review of photorejuvenation: devices, cosmeceuticals, or both? Dermatol Surg. 2005;31(9 Pt 2): 1166-1178.

5. Cosmeceuticals. US Industry Study with Forecasts for 2015 and 2020. Freedonia Group Inc., 2011. Available from: http://www.freedoniagroup. com/brochure/27xx/2758smwe.pdf. Accessed September 10, 2014.

6. Lavker RM, Kaidbey K, Leyden JJ. Effects of topical ammonium lactate on cutaneous atrophy from a potent topical corticosteroid. J Am Acad Dermatol. 1992;26(4):535-544.

7. Ramos-e-Silva M, Celem LR, Ramos-e-Silva S, Fucci-da-Costa AP. Anti-aging cosmetics: facts and controversies. Clin Dermatol. 2013; 31(6):750-758.

8. Rivers JK. The role of cosmeceuticals in antiaging therapy. Skin Therapy Lett. 2008;13(8):5-9.

9. Bernstein EF, Lee J, Brown DB, et al. Glycolic acid treatment increases type I collagen mRNA and hyaluronic acid content of human skin. Dermatol Surg. 2001;27(5):429-433.

10. Bissett DL, Miyamoto K, Sun P, et al. Topical niacinamide reduces yellowing, wrinkling, red blotchiness, and hyperpigmented spots in aging facial skin. Int J Cosmet Sci. 2004;26(5):231-238.

11. Bissett DL, Oblong JE, Berge CA. Niacinamide: a B vitamin that improves aging facial skin appearance. Dermatol Surg. 2005; 31(7 Pt 2):860-865.
12. Matts PJ, Oblong JE, Bissett DL. A review of the range of effects of niacinamide in human skin. IFSCC Magazine. 2002;5(4):285-289.

13. Fitzpatrick RE, Rostan EF. Double-blind, half-face study comparing topical vitamin $\mathrm{C}$ and vehicle for rejuvenation of photodamage. Dermatol Surg. 2002;28(3):231-236.

14. Sauermann K, Jaspers S, Koop U, Wenck H. Topically applied vitamin C increases the density of dermal papillae in aged human skin. $B M C$ Dermatol. 2004;4(1):13.

15. Mayer P. The effects of vitamin E on the skin. Cosmet Toiletr. 1993;108: 99-109.

16. Möller H, Ansmann A, Wallat S. The effects of vitamin E on the skin in topical applications. Fat Sci Technol. 1989;91(8):295-305.

17. Gassmueller J, Kecskés A, Jahm P. Stylus method for skin surface contour measurements. In: Serup J, Jemec GBE, editors. Handbook of Non-invasive Methods and the Skin. Boca Raton, FL: CRC Press; 1995:83-88.

18. O'goshi K. Suction cup method for measurement of skin mechanical properties: the cutometer. In: Serup J, Jemec GBE, editors. Handbook of Non-invasive Methods and the Skin. Boca Raton, FL: CRC Press; 1995:579-582.

19. Akhtar N, Zaman SU, Khan BA, et al. Calendula extract: effects on mechanical parameters of human skin. Acta Pol Pharm. 2011;68(5): 693-701.

20. Huang CK, Miller TA. The truth about over-the-counter topical antiaging products: a comprehensive review. Aesthetic Surg J. 2007;27(4): 402-412.

21. Dobrev H. In vivo study of skin mechanical properties in Raynaud's phenomenon. Skin Res Technol. 2007;13(1):91-94.

22. Koch RJ, Cheng ET. Quantification of skin elasticity changes associated with pulsed carbon dioxide laser skin resurfacing. Arch Facial Plast Surg. 1999;1(4):272-275.

23. Dobrev H. Use of Cutometer to assess epidermal hydration. Skin Res Technol. 2000;6(4):239-244.

24. Elson ML. Treatment of photoaging-a personal comment and open study of the use of glycolic acid. J Dermatol Treat. 1993;4(4):215-218.

25. Ditre CM, Griffin TD, Murphy GF, et al. Effects of $\alpha$-hydroxy acids on photoaged skin: a pilot clinical, histologic, and ultrastructural study. J Am Acad Dermatol. 1996;34(2 Pt 1):187-195.

26. Moy LS, Murad H, Moy RL. Glycolic acid therapy: evaluation of efficacy and techniques in treatment of photodamage lesions. Am J Cosmet Surg. 1993;10(1):9-13.

27. Rubino C, Farace F, Dessy LA, et al. A prospective study of anti-ageing topical therapies using a quantitative method of assessment. Plast Reconstr Surg. 2005;115(4):1156-1162; discussion 1163-1164.

28. Stiller MJ, Bartolone J, Stern R, et al. Topical $8 \%$ glycolic acid and $8 \%$ L-lactic acid creams for the treatment of photodamaged skin. A double-blind vehicle-controlled clinical trial. Arch Dermatol. 1996;132(6):631-636.

29. Smith WP. Epidermal and dermal effects of topical lactic acid. J Am Acad Dermatol. 1996;35(3 Pt 1):388-391.

30. Thibault PK, Wlodarczyk J, Wenck A. A double-blind randomized clinical trial on the effectiveness of a daily glycolic acid 5\% formulation in the treatment of photoaging. Dermatol Surg. 1998;24(5):573-577; discussion 577-578.

31. Zussman J, Ahdout J, Kim J. Vitamins and photoaging: do scientific data support their use? J Am Acad Dermatol. 2010;63(3):507-525.

32. Saurat JH, Didierjean L, Masgrau E, et al. Topical retinaldehyde on human skin: biologic effects and tolerance. J Invest Dermatol. 1994;103(6):770-774.

33. Dreno B, Fischer T, Perosino E, et al. Management of skin ageing: how to combine cosmetic procedures. Eur J Dermatol. 2008;18(4):444-451. 


\section{Publish your work in this journal}

Clinical, Cosmetic and Investigational Dermatology is an international, peer-reviewed, open access, online journal that focuses on the latest clinical and experimental research in all aspects of skin disease and cosmetic interventions. All areas of dermatology will be covered; contributions will be welcomed from all clinicians and basic science researchers globally. This journal is indexed on CAS. The manuscript management system is completely online and includes a very quick and fair peer-review system, which is all easy to use. Visit http://www.dovepress.com/testimonials.php to read real quotes from published authors.

Submit your manuscript here: http://www.dovepress.com/clinical-cosmetic-and-investigational-dermatology-journal 